\title{
Uso da Simulação Realística como Mediadora do Processo Ensino-Aprendizagem: Relato de Experiência da Farmácia Universitária da Universidade de São Paulo
}

\author{
Sílvia Storpirtis ${ }^{1 *}$, Maria Aparecida Nicoletti2*, Patrícia Melo Aguiar ${ }^{3 *}$ \\ 1,30partamento de Farmácia \\ 1,3Faculdade de Ciências Farmacêuticas da Universidade de São Paulo, ²Farmácia Universitária da Universidade \\ de São Paulo
}

* Autores para correspondência: sstor@usp.br, nicoletti@usp.br e aguiar.pm@usp.br

\begin{abstract}
RESUMO
O objetivo do presente artigo é apresentar a experiência da Farmácia Universitária da Universidade de São Paulo (Farmusp) no uso da simulação realística durante o Estágio Curricular Obrigatório em Práticas Farmacêuticas oferecido aos alunos da Faculdade de Ciências Farmacêuticas da USP (FCF-USP). Dentre as atividades desenvolvidas, os alunos elaboram um vídeo, no qual simulam uma situação clínica vivenciada durante o estágio, relacionada com o cuidado farmacêutico prestado aos pacientes atendidos na Farmusp. Essa atividade tem contribuído para a formação dos alunos, possibilitando o desenvolvimento de habilidades e atitudes.
\end{abstract}

Palavras-chave: Farmácia Universitária; Educação Farmacêutica; Simulação Realística.

\begin{abstract}
The aim of this study is to report the experience of the Universitary Pharmacy of University of São Paulo (Farmusp) in the use of realistic simulation during the Internship Required in Pharmaceutical Practices offered to students of the Faculty of Pharmaceutical Sciences of USP (FCF-USP). Among the activities, the students prepare a video in which they simulate a clinical situation experienced during the internship, related to pharmaceutical care provided to patients at Farmusp. This activity has contributed to the training of students, enabling the development of skills and attitudes.
\end{abstract}

Keywords: Universitary Pharmacy; Pharmaceutical Education; Realistic Simulation.

\section{Introdução}

A atual estruturação curricular da Faculdade de Ciências Farmacêuticas da Universidade de São Paulo (FGF-USP) foi concebida de acordo com a Resolução CNE/CES 02/2002, que instituiu as Diretrizes Curriculares Nacionais (DCN) dos Cursos de Graduação em Farmácia. Considerando a vocação da USP em desenvolver um ensino que acompanha as transformações das diversas áreas do conhecimento, em permanente diálogo com a sociedade, e a necessidade de integração entre o ensino, a pesquisa e a extensão universitária, a FGF-USP busca proporcionar formação abrangente, que atenda às diversas áreas de atuação do profissional farmacêutico. $\mathrm{O}$ modelo de ensino está apoiado no professor como facilitador e mediador do processo de ensino-aprendizagem, valorizando o desenvolvimento de competências (UNIVERSIDADE DE SÃO PAULO, 2016).

Segundo as DCN, a formação do farmacêutico deve contemplar as necessidades sociais na área da saúde, a atenção integral ao sistema regionalizado e hierarquizado de referência e contrarreferência e o trabalho em equipe, com ênfase no Sistema Único de Saúde. Os conteúdos essenciais para o Curso de Graduação em Farmácia devem estar relacionados ao processo saúde-doença do cidadão, da família e da comunidade, considerando a realidade epidemiológica e profissional (BRASIL, 2002; CONSELHO FEDERAL DE FARMÁGIA, 2008). 
Nesse sentido, a FCF-USP iniciou o processo de readequação da Farmácia Universitária da Universidade de São Paulo (Farmusp), campus capital, criada em 1970 e que integra o Centro de Controle de Medicamentos e Assistência Farmacêutica do Departamento de Farmácia (CCAF). O novo modelo, praticado desde 2008, visa à formação do aluno considerando a interdisciplinaridade em saúde, tendo o paciente como foco principal e o medicamento como insumo aplicável ao seu tratamento (modelo biopsicossocial).

Essa formação integral, segundo as recomendações nacionais e internacionais, requer que o farmacêutico atue no sistema de saúde como membro de uma equipe multiprofissional, pautado pela prática da farmácia clínica e da atenção farmacêutica (ou cuidado farmacêutico), visando não só a melhorar a qualidade de vida do usuário, mas também a realizar os objetivos de saúde do sistema como um todo (MARIN et al., 2003; STORPIRTIS et al., 2008; ROVERS \& CURRIE, 2010; STORPIRTIS et al., 2011).

Considerando a importância da universidade na formação profissional, particularmente no ensino farmacêutico, torna-se fundamental que os profissionais sejam preparados para o enfrentamento do cenário real de saúde pública no Brasil, com desenvolvimento de análise crítica para a prática da gestão e a tomada de decisão em saúde. Também se deve superar o desafio de aproximar a academia e os serviços de saúde e de conciliar as atividades de ensino, pesquisa e assistência (CASTILHO \& ELIAS, 2014; LEITE \& MANZINI, 2015; STORPIRTIS et al., 2016).

Segundo o Conselho Federal de Farmácia, a Farmácia-Escola (atualmente denominada Farmácia Universitária) representa um cenário de prática essencial, além de elemento determinante na qualidade do processo de ensino-aprendizagem, sendo também considerada um indicador de qualidade dos cursos de Farmácia (CONSELHO FEDERAL DE FARMÁCIA, 2008; MINISTÉRIO DA EDUCAÇÃO, 2015).

No curso de graduação de Farmácia-Bioquímica da FCF-USP estão previstos dois está- gios obrigatórios com duração total mínima de novecentas horas (17\% da carga horária total do curso). O estágio denominado Práticas Farmacêuticas tem duração de 120 horas e pode ser realizado a partir do quarto semestre, nos períodos integral e noturno. O programa deve ser desenvolvido sob a orientação de um profissional farmacêutico em Unidades de Assistência Médica Ambulatorial (AMA), Unidades Básicas de Saúde (UBS), Hospitais, Farmácias ou em Ambulatório Médico de Especialidades (AME). Já o estágio em Atividades Farmacêuticas tem duração de 780 horas e pode ser realizado a partir do sétimo semestre do período integral e do nono semestre do período noturno e também inclui a Assistência e Atenção Farmacêuticas, além de outras áreas (UNIVERSIDADE DE SÃO PAULO, 2016).

Nesse contexto, a Farmusp disponibiliza os estágios Práticas Farmacêuticas e Guidados Farmacêuticos, em complexidades diferenciadas, para estimular a análise crítica de situações de baixa, média e alta complexidade vivenciadas, com a proposição de soluções baseadas em evidência científica, desafiando, assim, a capacidade criativa do estagiário por meio de divulgação de informação em saúde, em linguagem adequada, utilizando ferramentas de tecnologia da informação.

Dentre vários fatores, a formação profissional adequada é uma das responsáveis pelo sucesso de quaisquer atividades desenvolvidas na área da saúde, considerando que, em todos os níveis de atuação, será necessária formação sólida para a prática das habilidades e competências inerentes às diferentes profissões (CORDEIRO \& LEITE, 2005; COLLENDER, 2009; LYRA JR. \& MARQUES, 2012).

Cabe ressaltar que o ensino é essencial para a inovação de serviços e produtos, fato que a FCF-USP reconhece como fundamental e que norteou a concepção do modelo inovador no atendimento ao paciente desenvolvido na Farmusp, disponibilizando aos alunos e à comunidade ações de vanguarda que assegurem o uso racional de medicamentos, objetivando, ainda, a 
humanização no cuidado ao paciente. Tanto alunos quanto docentes estão atentos às necessidades de atualização da grade curricular do curso para a formação do farmacêutico e, portanto, a graduação deve cumprir seu papel de relevância no entendimento das necessidades da sociedade em relação à saúde (MORIN, 2000; BRASIL, 2001; CONSELHO FEDERAL DE FARMÁCIA, 2008).

\section{Objetivo}

Relatar a experiência da Farmusp no uso da simulação realística durante o estágio curricular realizado por acadêmicos do curso de Farmácia-Bioquímica da FCF-USP, campus capital.

\section{Desenvolvimento}

O ensino deve capacitar o recém-formado para a entrada no mercado de trabalho, de modo a que sua atuação profissional seja facilitada, trazendo, portanto, sua colaboração ativa para a proposição de soluções e/ou ações para a resolução de problemas que afetam a comunidade.

Normalmente, pressupõe-se que a inteligência seja uma faculdade que torna possível o acúmulo e o armazenamento de informação e, nesse sentido, a sua transmissão faz parte da educação formal. Entretanto, as gerações de alunos que se seguem são apresentadas a novas ferramentas de ensino-aprendizagem evitando, assim, a perpetuação de modelos automatizados, tornando o aprender uma atividade facilitada com a linguagem de cada geração (GATTI, 2013). O ensino na universidade constitui-se em processo de busca, de construção científica e de crítica ao conhecimento produzido, ou seja, ao seu papel na construção da sociedade, com atribuições relacionadas, por exemplo, ao conhecimento do universo cultural dos alunos, desenvolvendo processos de ensino-aprendizagem interativos e participativos (PIMENTA \& ANASTASIOU, 2002).

Pesquisadores e docentes do campo da saúde notaram a necessidade de aprimoramento das técnicas de ensino. Novas teorias relacionam-se com técnicas que envolvem situações vivenciadas, de forma mais fidedigna e realística. Assim, a simulação teve seu conceito tradicional reformulado, sendo reco- nhecida como um conjunto de técnicas elaboradas com o intuito de recriar, de forma substitutiva e ampla, um espaço laboral e terapêutico que permita a participação do aprendiz (GABA, 2004).

A simulação realística tem sido bastante empregada como estratégia de ensino, treinamento e avaliação prática de habilidades em diversos cursos da saúde (COUTO, 2014). Como ferramenta de treinamento inovadora e sofisticada, utiliza recursos como simuladores de pacientes, atores profissionais e outros personagens para a representação de situações cotidianas e reais em uma área física apropriada, contextualizada na atividade específica proposta, onde os partícipes podem interagir em algum momento, além de poder gerar a discussão em grupos sobre a ação demonstrada (debriefing) (ALBERT EINSTEIN INSTITUTO ISRAELITA DE ENSINO E PESQUISA, 2016).

De modo a disponibilizar a linguagem de tecnologia da informação como instrumento de aprendizado aos alunos da FCF-USP, o Programa de Estágio Curricular desenvolvido na Farmusp foi reformulado e, desde janeiro de 2015, incluiu a elaboração de um vídeo que apresente a simulação de uma situação clínica vivenciada durante o estágio, relacionada com o cuidado farmacêutico oferecido aos pacientes. Para tanto, disponibiliza-se infraestrutura para a montagem dos diferentes cenários de simulação e realização de filmagem, incluindo a questão da interdisciplinaridade. Nesses cenários, cada novo grupo de estagiários tem à disposição materiais variados e equipamentos eletroeletrônicos que possibilitam a representação de situações reais por eles criadas. $\mathrm{O}$ material desenvolvido passa a integrar a videoteca da Farmusp (atualmente com dez vídeos) ou é disponibilizado na internet (quatro vídeos já constam no YouTube), constituindo um material didático-instrucional para os próximos grupos de estagiários.

Os casos simulados pelos estagiários envolvem pacientes com doenças crônicas e problemas de adesão ao tratamento, pacientes analfabetos ou com baixa escolaridade, com ou sem cuidador, presença de interações medicamentosas, comorbidades, entre outros (Figura 1). O consultório farmacêutico (sala de atendimento) de uma unidade de saúde ou 
farmácia é o cenário de prática mais utilizado pelos alunos, onde ocorre a simulação da prestação do serviço de acompanhamento farmacoterapêutico a pacientes ambulatoriais (Figura 2).

Também merece destaque o cenário referente ao serviço de dispensação de medicamentos (Figura 3). Os atores (estagiários) estruturam um fluxo de situações de maneira a possibilitar a aplicação de condutas éticas no manejo do binômio doença-paciente. $\mathrm{A}$ duração dos vídeos é variada, diretamente relacionada à complexidade da situação e ao desfecho proposto.

Os roteiros para as simulações criadas pelos alunos são discutidos com o grupo desenvolvedor em consenso com o orientador do estágio, de maneira que o tema vá ao encontro dos anseios dos alunos em relação às ideias propostas. Ao final do estágio, os graduandos apresentam o vídeo desenvolvido para a equipe de profissionais presentes na Farmusp, colegas, professores e demais estagiários, gerando discussão acerca das situações contextualizadas (Figura 4).

\section{Conclusão}

O processo de ensino-aprendizagem no ensino superior deve evoluir paralelamente às necessidades sociais, e o desenvolvimento de competências e habilidades tem sido considerado fundamental

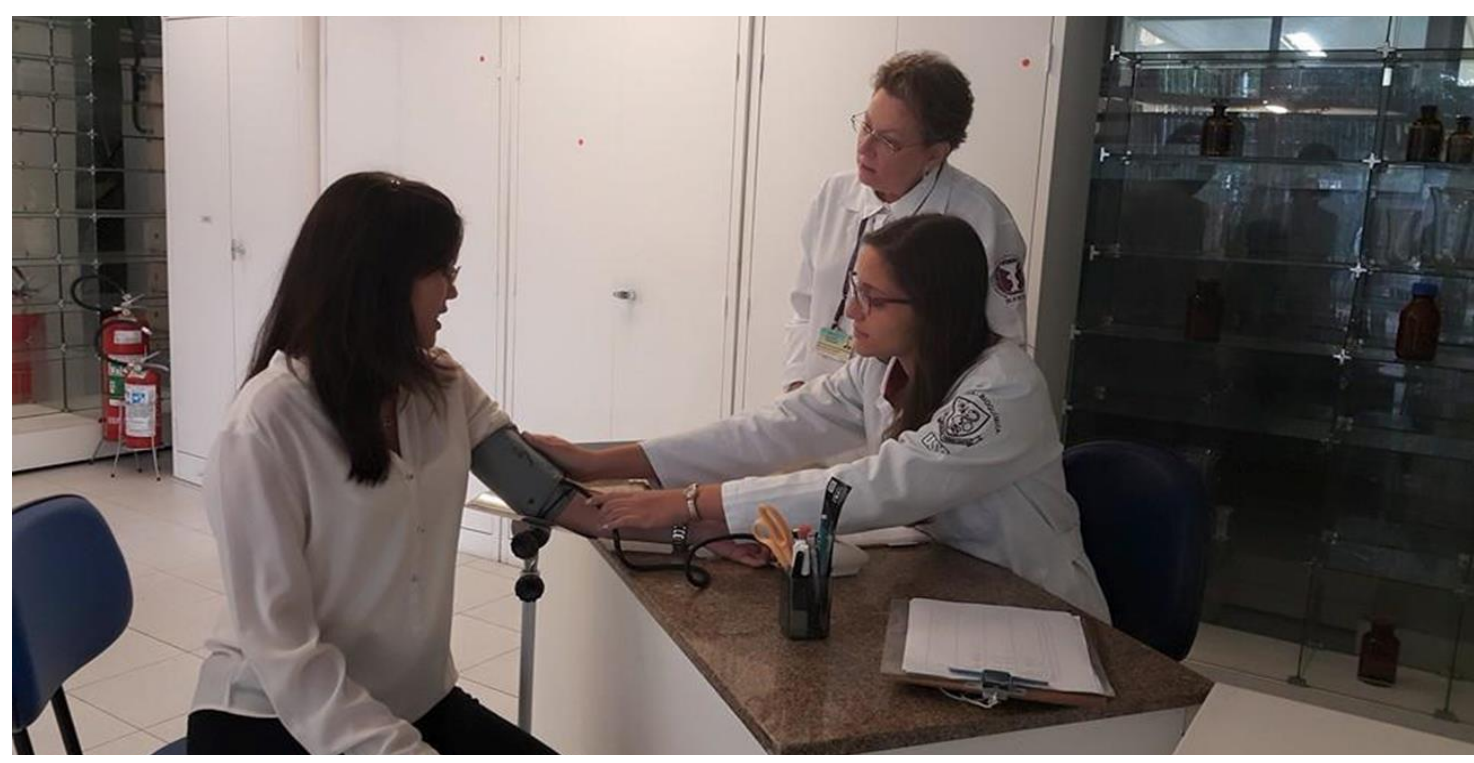

Figura 1 - Simulação realística entre farmacêutico e paciente. Fonte: Farmusp.

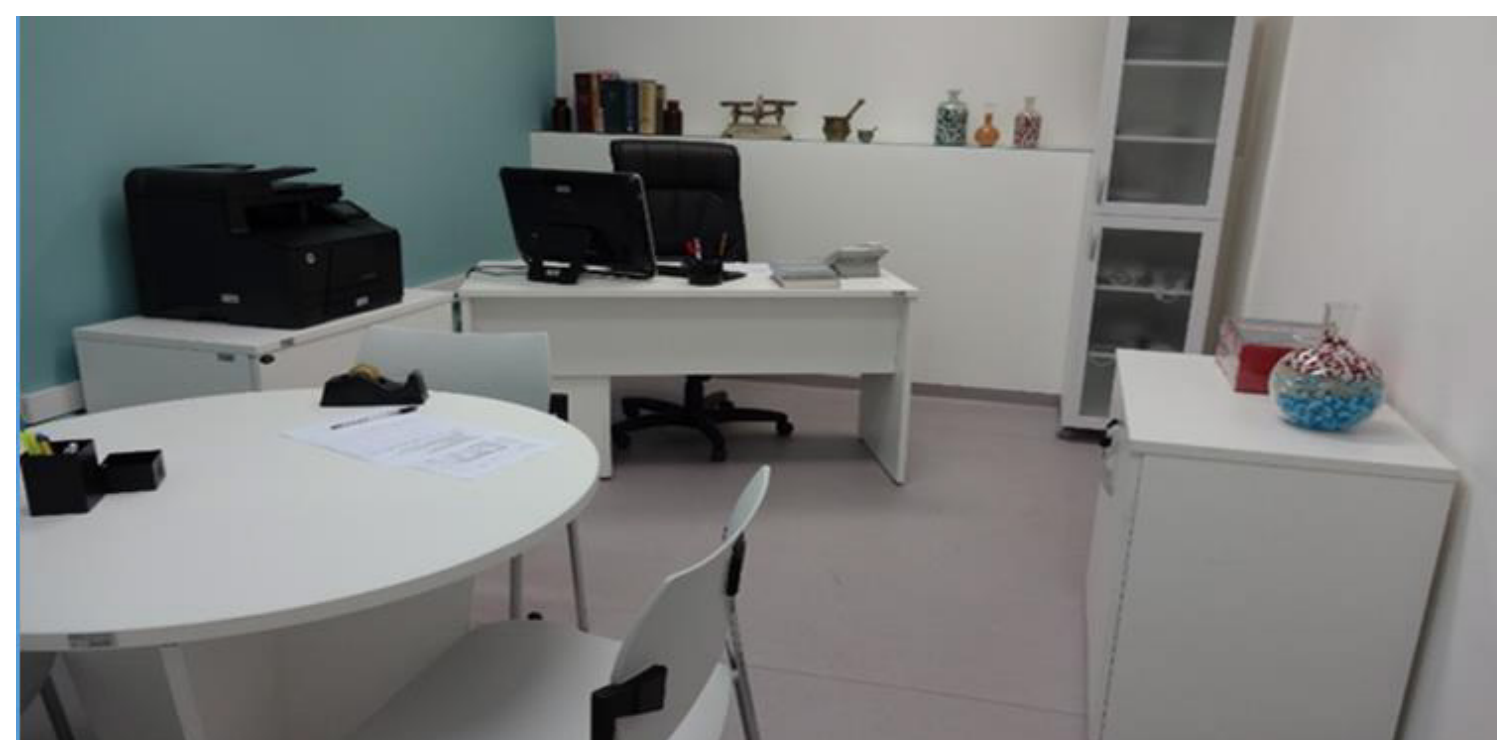

Figura 2 - Área de simulação realística relacionada ao serviço de acompanhamento farmacoterapêutico a pacientes ambulatoriais (consultório farmacêutico ou sala de atendimento). Fonte: Farmusp. 


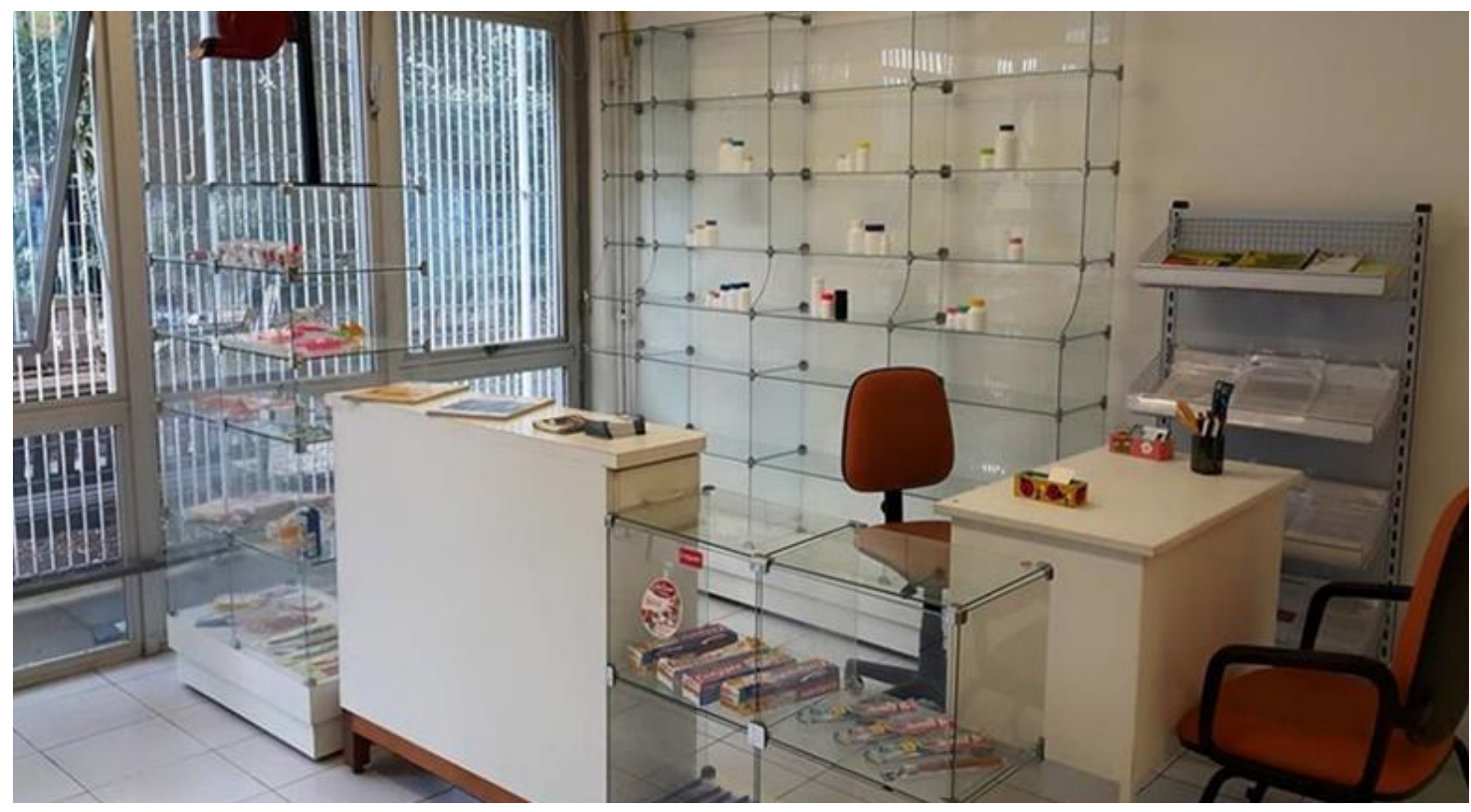

Figura 3 - Área de simulação realística de farmácia como estabelecimento de saúde para treinamento envolvendo diferentes atores (profissionais e usuários de medicamento). Fonte: Farmusp.

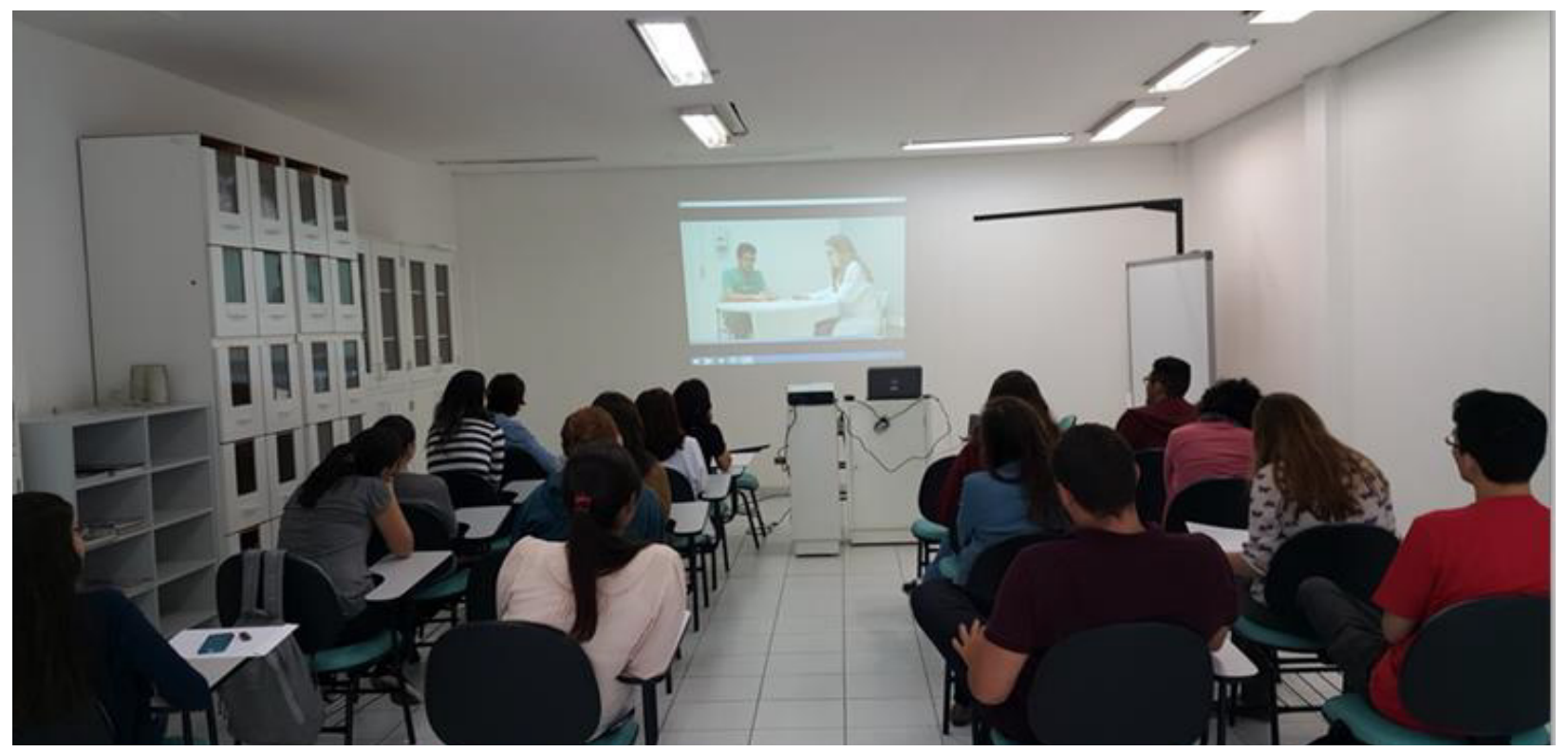

Figura 4 - Apresentação de vídeo elaborado pelos alunos durante o estágio na Farmusp. Após a projeção, há realização do debriefing (discussão das situações levantadas). Fonte: Farmusp.

para que os graduandos na área de Farmácia compreendam e estejam preparados para atuar na resolução de problemas.

Por meio da elaboração de vídeos durante a realização do estágio curricular na Farmusp, os alunos da FGF-USP demonstram criatividade e o desenvolvimento de habilidades e atitudes com o uso de simulação realística, resultando na produção de mídia digital com abordagem educacional, fruto da experiência adquirida.
Ressalta-se que há intensa motivação dos estudantes nesse processo, o que contribui para a construção de conhecimento embasado em comportamento ético, inserido nas práticas relacionadas com a prestação do cuidado farmacêutico a pacientes atendidos na Farmusp.

Pelo exposto, cabe destacar o texto de Paulo Freire (2002, p. 21): "Ensinar não é transferir conhecimento, mas criar possibilidades para a sua produção ou sua construção". 


\section{Referências Bibliográficas}

ALBERT EINSTEIN INSTITUTO ISRAELITA DE ENSINO E PESQUISA. Centro de Educação em Saúde Abram Szajman. "Atualização Profissional: Metodologia da Simulação Realística: Por Onde Começar?", 2016. Disponível em: \http://www.einstein. $\mathrm{br} /$ Ensino/cursos-de-atualizacao/Paginas/curso-deatualizacao-em-metodologia-da-simulacao-realistica-poronde-comecar.aspx $>$. Acessado em: 24 out. 2016.

BRASIL. Ministério da Saúde. Secretaria de Políticas de Saúde. Departamento de Formulação de Políticas de Saúde. Politica Nacional de Medicamentos. Brasília: Ministério da Saúde, 2001. 40p. (Série C. Projetos, Programas e Relatórios, n. 25).

BRASIL. "Resolução GNE/CESU n. 2/02 de 19 de fevereiro de 2002". Institui diretrizes curriculares nacionais do curso de graduação em Farmácia. Diário Oficial da União. Brasília, Seção 1, 4 mar. 2002, p. 9.

CASTILHO, Selma R. \& ELIAS, Sabrina G. "Importância da Aproximação entre Academia e Serviços de Saúde e o Desafio de Conciliar Ensino, Pesquisa e Assistência". In: CARVALHO, Felipe D.; CAPUCHO, Helaine C.; BISSON, Marcelo P. Farmacêutico Hospitalar: Conhecimentos, Habilidades e Atitudes. São Paulo: Manole, 2014, pp. 27-34.

COLLENDER, Guy. "The Rise of Interdisciplinary Approaches: How Scientists Are Working Together for International Development". International Pharmacy fournal - IPF, The Official Fournal of FIP, vol. 24, n. 1, jun. 2009, pp. 4-9. Disponível em: \http://fip.org/files/ fip/IPJ/IPJ_2009V24_DEFweb.pd† $>$. Acessado em: 24 out. 2016.

CONSELHO FEDERAL DE FARMÁCIA (org.). FERNANDES, Zilamar G.; BERMOND, Magali D.; OLIVEIRA-FILHO, Nilsen C. F.; CAMARGO, Ely E. S. \& CECY, Carlos. Os Desafios da Educaşão Farmacêutica no Brasil. Brasília: Conselho Federal de Farmácia, 2008. 131p.

CORDEIRO, Benedito C. \& LEITE, Silvana N. O Farmacêutico na Atenşão à Saúde. Itajaí: Universidade Vale do Itajaí, 2005.

COUTO, Thomas B. Simulação Realística no Ensino de Emergências Pediátricas na Graduação. Dissertação (Mestrado). Faculdade de Medicina da Universidade de São Paulo, 2014.

FREIRE, Paulo. Pedagogia da Autonomia. Saberes Necessários à Prática Educativa. 25. ed. São Paulo: Paz e Terra, 2002.

GABA, David. "The Future Vision of Simulation in Health Care". Quality \& Safety Health Care, vol. 13, n. 1, 2004, pp. 2-10.

GATTI, Bernadete A. "A Prática Pedagógica como Núcleo do Processo de Formação de Professores". In: GATTI, Bernadete A.; SILVA-JÚNIOR, Celestino
A. S.; PAGOTTO, Maria D. S.; NICOLETTI, Maria G. (orgs.). Por uma Politica Nacional de Formação de Professores. São Paulo: Editora Unesp, 2013, pp. 95-106.

LEITE, Silvana N. \& MANZINI, Fernanda. "Estruturação da Assistência Farmacêutica", capítulo 3. In: CONSELHO FEDERAL DE FARMÁCIA. Grupo de Trabalho sobre Saúde Pública. O Farmacêutico na Assistência Farmacêutica do SUS: Diretrizes para Ação. Brasília: Conselho Federal de Farmácia, 2015, pp. 50-67.

LYRA JÚNIOR, Divaldo \& MARQUES, Tatiane C. As Bases da Dispensação Racional de Medicamentos para Farmacêuticos. São Paulo: Pharmabooks, 2012. 270p.

MARIN, Nelly; LUIZA, Vera L.; OSORIO-DECASTRO, Claudia G. S.; MACHADO-DOS-SANTOS, Sílvio (orgs.). Assistência Farmacêutica para Gerentes Municipais. Rio de Janeiro: Opas/OMS, 2003. 336p.

MINISTÉRIO DA EDUCAÇÃO. Instituto Nacional de Estudos e Pesquisas Educacionais Anísio Teixeira - Inep. Diretoria de Avaliação da Educação Superior - Daes. "Nota Técnica Daes/Inep n. 023/2015, de 8 de junho de 2015. Esclarecimentos sobre o indicador 3.6 do Instrumento de Avaliação do Curso de Graduação Presencial e a Distância". Disponível em: <http://download.inep.gov.br/educacao_superior/ avaliacao_cursos_graduacao/legislacao_normas/2015/ nota_tecnica_DAES-INEP_n23_2015.pdP. Acessado em: 24 out. 2016.

MORIN, Edgar. Complexidade e Transdisciplinaridade: a Reforma da Universidade e do Ensino Fundamental. Natal: EDUFRN, 2000.

PIMENTA, Selma G. \& ANASTASIOU, Lea G. C. Docência no Ensino Superior. São Paulo: Cortez, 2002. 279p. vol. 1: Docência em Formaşão. Ensino Superior.

ROVERS, John P. \& CURRIE, Jay D. A Practical Guide to Pharmaceutical Care: a Clinical Skills Primer. 3rd ed. Washingd ton: American Pharmacists Association, 2007. 259 p.

STORPIRTIS, S.; NELLA GAI, María; DE CAMPOS, Daniel R. \& GONÇALVES, José E. Farmacocinética: Básica e Aplicada. Rio de Janeiro: Guanabara Koogan, 2011. 240p.

STORPIRTIS, Sílvia; FERREIRA, Elisabeth I. \& NICOLETTI, Maria A. "Bases Conceituais do Novo Modelo de Atuação da Farmácia Universitária da Universidade de São Paulo (Farmusp)". 2016. Disponível em: <http://www.fcf. usp.br/arquivos/departamentos/FARMUSP/ Bases $\% 20$ Conceituais $\% 20$ do $\% 20$ Modelo $\% 20$ inovador $\% 20 \mathrm{da} \% 20$ FARMUSP_Farmácia $\% 20$ Universitária \%2 0da\%20FCF-USP_19.05.2015. pdf $>$. Acessado em: 24 out. 2016.

STORPIRTIS, Sílvia; MORI, Ana L. P. M.; YOCHIY, Angélica; RIBEIRO, Eliane \& PORTA, Valentina. Farmácia Clínica e Atenção Farmacêutica. Rio de Janeiro: Guanabara Koogan, 2008. 489p. 
UNIVERSIDADE DE SÃO PAULO. Faculdade de fcf.usp.br/graduacao/pagina.php?menu=51\&pagina=408> Ciências Farmacêuticas. "Projeto Político Pedagógico do Curso Acessado em: 24 out. 2016 de Farmácia-Bioquímica". 2016. Disponível em: \http://www.

Publicado em 11/11/2016. 